\title{
Transferencia morfosintáctica del aimara en el castellano
}

\author{
Miguel Angel Romero Sihuayro ${ }^{1 \mathrm{a}}$ \\ Facultad de Ciencias Humanas y Educación, Universidad Peruana Unión ${ }^{1}$ \\ Orcid ID: https://orcid.org/0000-0002-8735-94241
}

Recibido: 13 de noviembre de 2017

Aceptado: 28 de diciembre de 2017

\section{Resumen}

Esta investigación es de enfoque cualitativo de tipo descriptivo, se centró en describir la trasferencia morfosintáctica del aimara en el castellano en los estudiantes del quinto grado de la Institución Educativa Primaria N 70274 Challapampa - Copani - Yunguyo - Puno, 2017. La muestra la conforman 12 estudiantes del quinto grado sección única, elegidos por tipo de muestreo intencional no probabilístico; para el recojo de datos se aplicó dos fichas: La primera denominada "Ficha de Desempeño de Competencia Lingüística en la Producción de Textos Escritos en Castellano" y la segunda "Ficha de Desempeño de Competencia Lingüística en la Producción de Textos Orales en Castellano", los datos se analizaron con la técnica de Análisis Contrastivo de Corder. El resultado de la investigación, muestra que el contacto de lenguas que caracteriza al C.P. Challapampa, da lugar a fenómenos de trasferencia lingüística especialmente, en el nivel morfosintáctico donde los estudiantes hacen uso de la estructura morfosintáctica propia del aimara $\mathrm{S}+\mathrm{O}+\mathrm{V}$ para producir textos escritos en castellano, lo cual es incorrecto, puesto que la estructura del castellano es $\mathrm{S}+\mathrm{V}+\mathrm{O}$; también se evidenció el uso frecuente de expresiones como hemos y taba; además de préstamos lingüísticos del idioma aimara como las expresiones waka, awatiw; por otra parte se aprecia el reemplazo de vocales como la /e/ por la /i/.

Palabras clave: Trasferencia linguística; morfosintáxis; competencias linguísticas; lengua materna.

\section{Morphosyntactic transference of Aymara in Spanish}

\author{
Abstrac

This research study uses a descriptive type of qualitative approach, focused on describing the morphosyntactic transference of Aymara in Spanish among students of the fifth grade in the Primary School No 70274 Challapampa - Copani - Yunguyo - Puno, 2017. The sample was made up of 12 students from the only fifth grade classroom, chosen by intentional non- 
probabilistic sampling. Two instruments were applied to collect the data: The first one called "Linguistic Competency Performance Record in the Production of Written Texts in Spanish" and the second "Linguistic Competency Performance Report in the Production of Spanish Oral Texts", the data were analyzed with the Corder Contrastive Analysis technique. The result of the research show that the contact of languages which characterizes the C.P. Challapampa, gives rise to linguistic transference phenomena, especially at the morphosyntactic level where students make use of the morphosyntactic structure of Aymara $\mathrm{S}+\mathrm{O}+\mathrm{V}$ to write in Spanish, which is incorrect, since the structure of Spanish is $\mathrm{S}+\mathrm{V}+\mathrm{O}$; frequent use of expressions such as hemos and taba; loan words from the Aymara language such as the expressions waka, awatiw; and finally the replacement of the vowel/e/ with /i/.

Key-words: linguistic transference; morphosyntaxis; language skills; mother tongue

\section{Introducción}

La humanidad se comunicaba a través de una sola lengua o idioma, aunque no se menciona cuál era, así se refiere en Génesis 11:1 donde se menciona que "Había entonces en toda la tierra una sola lengua y unas mismas palabras".

Posteriormente cuando los hombres empezaron a construir la torre de Babel, el señor dijo: "Ahora pues, descendamos y confundamos allí su lenguaje" (Génesis 11:7). De esa forma se crearon los diferentes idiomas y grupos lingüísticos existentes en la humanidad.

En el Perú, según Miranda (1998), hasta la conquista española en 1532 se desconocía la real situación linguiística del antiguo Perú, pero gracias a Cerrón Palomino y Robledo podemos estimar que la mayor parte del Tahuantinsuyo era de habla quechua o runa - simi, la segunda lengua originaria y antecesora del quechua era el jaqi aru hoy conocida como aimara y finalmente la tercera lengua el pukina. Por lo que deducimos que las lenguas quechua y aimara eran las más difundidas. Hasta el siglo XVIII la situación de todos los grupos étnicos existentes en el Perú cambiaron, presentándose la hegemonía del castellano; hoy en día en el Perú conviven muchas lenguas ancestrales existiendo alrededor de 40 lenguas aproximadamente. En las últimas décadas, según Robledo (2008) "las políticas lingüísticas en el Perú, que deberían buscar la integración y asegurar el progreso de sociedades sumamente diversas que comparten un territorio como el nuestro, reflejan claramente, por el contrario, una situación de dominio del castellano que se ha consolidado con el trascurso de los siglos” (p. 77).

El objetivo primordial del presente artículo de investigación es describir la trasferencia morfosintáctica del aimara en el castellano en los estudiantes de la Institución Educativa Primaria $\mathrm{N}^{\circ} 70274$ Challapampa, provincia de Yunguyo, Puno; especialmente en la oración simple conformada por un sujeto, verbo y objeto o complemento ya que la influencia del aimara y el castellano es mutua en los niños y jóvenes puneños de habla aimara y castellana, pero en 
este caso solo se aborda la trasferencia morfosintáctica de mayor uso en los textos escritos y orales de los estudiantes bilingües. Este problema amerita seguir investigándose para contrarrestar las falencias, que se presentan en la producción de textos en español por la influencia que ejerce el aimara.

Tratar sobre el idioma es un tema muy complejo, por lo que es necesario tener una clara definición de este término, para la Real Academia Española, idioma es "la lengua de un pueblo, nación o común a varios"; el idioma posee una gramática y sus reglas correspondientes y si hablamos de un sistema hablamos también de una estructura y este último se conoce como morfosintaxis que viene a ser la estructura, la forma y el sentido que presenta cada idioma. Antes de poder describir debemos comprender plenamente el término sintaxis, para muchos investigadores es básicamente la forma cómo se estructura una oración en una determinada lengua o idioma, así la Real Academia Español (1998) define como sintaxis el "análisis de las estructuras que se crean en función de la forma en que se ordenan y se combinan las palabras"; teniendo en claro estos términos podemos comprender la presente investigación.

Morfosintaxis del castellano: Según la Real Academia Española (1998) la morfosintaxis de la lengua castellana en una oración simple es conformada por Sujeto + Verbo + Objeto o Complemento (S V O), y el orden como se estructura es así:

Ejemplo: Las estrellas brillan en la noche.

\section{Sujeto Verbo Objeto}

- Sujeto

- Verbo

- Objeto o complemento
: Las estrellas

: brillan

: en la noche.

Observamos que la lengua castellana posee una estructura particular para estructurar el sujeto, verbo y el objeto o complemento (S V O), lo que es visible en una oración simple.

Morfosintaxis del aimara: Según Bertonio (1612) la morfosintaxis de la lengua aimara en una oración simple está conformada por Sujeto + Objeto o Complemento + Verbo (S O V), y es así el orden como se estructura:

Ejemplo: Warawaranakax $\underline{\text { arumanw }} \underline{k^{\prime} a j i}=$ Las estrellas brillan en la noche.

\section{Sujeto Objeto Verbo}

- Sujeto

- Objeto o complemento

- Verbo
: Warawaranakax $=$ Las estrellas.

: arumanw = en la noche.

: $k^{\prime}$ aji = brillan. 
Merma (2007, p. 192) refuerza lo expuesto:

el castellano es una lengua flexible, y el aimara es una lengua aglutinante y, mientras que el castellano coloca el objeto de manera predominante después del verbo ( $\mathrm{S}$ V O), el aimara lo coloca antes ( $\mathrm{S} \mathrm{O}$ $\mathrm{V})$. Desde el punto de vista sociolingüístico, el castellano y el aimara representa diferentes tipos de comunidades lingüísticas.

\section{Contacto de lenguas}

Desde que el hombre interactuó con su semejante ya se dio el contacto de lenguas, lo que hoy es más notorio con la globalización, rompiendo todas las barreras; por eso se estudia las características del español andino como consecuencia del contacto de lenguas entre el castellano y el aimara en el territorio peruano y sobre todo en la región Puno. La región de Puno se caracteriza por ser una zona quechua - aimara, la mayor parte de su población es bilingüe, es evidente que el territorio peruano está conformada por diversos grupos étnicos que poseen su propia lengua y cultura. Es así que el Centro Poblado de Challapampa presenta su propia peculiaridad de cultura y lengua, donde sus pobladores son de habla aimara bilingües.

Weinreich (1974, p. 17) uno de los pioneros en el estudio de las lenguas en contacto, declara que "dos o más lenguas están en contacto si son usadas alternativamente por las mismas personas, y los individuos que usan las lenguas son, por tanto, el punto de contacto". Así mismo Limo y Salcedo (2008) indica:

el contacto de lenguas es una situación en la que dos o más lenguas conviven en un mismo espacio que involucran no solamente factores lingüísticos, sino también políticos, económicos y sociales, debido a que no se pueden desligar las lenguas de aquellos que las hablan (p. 127).

\section{Consecuencias del contacto de lenguas}

Para Zimmermann (2004) las consecuencias del contacto de lenguas traen consigo la diglosia y el bilingüismo. Por su parte Cerrón (2003), manifiesta que donde hay lenguas en contacto es normal encontrar problemas de trasferencia que afectan la perfomance de los bilingües.

Afirmamos entonces que la trasferencia es una consecuencia del contacto de lenguas.

\section{La trasferencia lingüística}

La trasferencia lingüística para muchos autores es definida como préstamo, cambio linguiístico, desviación de la norma estándar. Es así como Granda (1994), la precisa como "los fenómenos de modificación de estructuras originados por el influjo de una lengua sobre otra, en contacto con ella"

Algunas de las investigaciones sobre la trasferencia lingüística en nuestro país son las siguientes: 
La investigación desarrollada por Cortez (1999) en torno a las "Características lingüística, culturales y educativas que presentan los estudiantes indígenas que ingresaron a la Universidad Mayor de San Marcos en 1999", destaca que los estudiantes presentan dificultades en el uso del castellano, tanto en forma oral como escrita.

Benavente (1985), en su tesis: "El castellano en Puno: Variaciones sintácticas", refiere que observó un castellano "motoso", donde se hace excesivo uso de diminutivos y el uso del elemento narrativo "dice".

Godenzzi (2005) en su libro: "En las redes del lenguaje", señala que los rasgos peculiares del castellano andino es muchas veces discriminado y catalogado como "motoso".

En síntesis la estructura particular del idioma aimara: $\mathrm{S}+\mathrm{O}+\mathrm{V}$ se trasfiere al momento de hablar o escribir en castellano, no respetando la estructura de esta última lengua: $\mathrm{S}+\mathrm{V}+\mathrm{O}$.

\section{Materiales y Métodos}

La investigación presenta un enfoque cualitativo de tipo descriptivo, porque se describe la transferencia morfosintáctica del aimara en la producción de textos escritos y orales en castellano.

\subsection{Participantes}

Los participantes son 12 estudiantes elegidos por tipo de muestreo intencional no probabilístico, quienes pertenecen al quinto grado de la Institución Educativa Primaria $\mathrm{N}^{\circ}$ 70274 Challapampa - Copani - Yunguyo - Puno, 2017. La edad de dichos estudiantes está comprendida entre 10 y 11 años de edad, siendo una institución mixta lo conforman varones y mujeres; al residir en el Centro Poblado de Challapampa todos ellos son estudiantes bilingües aimara - castellano.

\subsection{Instrumentos}

Los instrumentos empleados fueron la "Ficha de Desempeño de Competencia Lingüística en la Producción de Textos Escritos en Castellano", validado y ejecutado por Montalvo (2014). Esta ficha comprende tres partes, la primera recoge datos demográficos, la segunda pide que se registre cinco oraciones simples, y la tercera solicita que puedan escribir una experiencia o anécdota; todas estas en el idioma castellano.

La segunda ficha empleada es la "Ficha de Desempeño de Competencia Lingüística en la Producción de Textos Orales en Castellano”, esta ficha registra la trascripción de la entrevista 
oral que se realizó a los estudiantes encuestados, sólo se realizó una pregunta ¿Qué hiciste en vacaciones?, donde los estudiantes son grabados.

\subsection{Análisis de datos}

De las 12 fichas de desempeño de competencias lingüísticas en la producción de textos escritos en castellano por los estudiantes bilingües (aimara - castellano) de la Institución Educativa Primaria $N^{\circ} 70274$ Challapampa, se han seleccionado 03 fichas en las cuales se observan fenómenos de trasferencia morfosintáctica, y sus respectivas fichas de desempeño de competencias lingüísticas en la producción de textos orales en castellano.

El análisis de los datos obtenidos se realizó con la técnica de Análisis Contrastivo de Corder, aplicada por Rodríguez y Tovar (1993); el procedimiento establecido para este análisis contrastivo es:

1. Selección de Corpus: El corpus que se analiza está constituido por los enunciados de los estudiantes, en las que se identifican los errores por trasferencia morfosintáctica.

2. Identificación de los errores: Se identifica solo los errores cometidos por sintaxis del aimara en la producción de textos escritos en castellano.

3. Clasificación, explicación y conclusión: En este artículo se ha clasificado los errores de trasferencia según criterios gramaticales. Para la explicación de los errores, se recurre a la gramática aimara para determinar el origen de la interferencia o trasferencia. La conclusión constituye el análisis final de la trasferencia lingüística.

\section{Resultados y discusión}

El objetivo general del presente artículo científico es describir la trasferencia morfosintáctica del aimara en la producción de textos escritos y orales en el castellano, en los estudiantes del quinto grado de educación primaria. Los doce estudiantes bilingües que conforman la muestra, residen en el área rural del C.P. Challapampa. En su tiempo libre se dedican al cuidado de animales domésticos (vacunos, ovinos, porcinos, avícolas y otros animales domésticos), cuyas experiencias se registran en los textos escritos y orales que produjeron.

\subsection{Resultados 1: Datos generales de información}

A continuación, se presenta los datos generales de información de los estudiantes del quinto grado sección única, seleccionados para el presente estudio. 


\section{Tabla 1}

Datos generales de información de los estudiantes de la Institución Educativa Primaria $N^{\circ}$ 70274 Challapampa

\begin{tabular}{|c|c|c|c|c|c|c|}
\hline \multirow[t]{2}{*}{ EST } & \multicolumn{3}{|c|}{$\begin{array}{c}\text { Datos } \\
\text { Personales } \\
\end{array}$} & \multirow[t]{2}{*}{ Estudiante } & \multirow[t]{2}{*}{ Padres } & \multirow{2}{*}{ Abuelos } \\
\hline & Edad & Grado & Sexo & & & \\
\hline Est. 03 & 10 & 5 to & $\mathrm{F}$ & $(\mathrm{L} 1)(\mathrm{L} 2)$ & (L1)(L2) & (L1) \\
\hline Est. 09 & 11 & 5 to & $\mathrm{F}$ & $(\mathrm{L} 1)(\mathrm{L} 2)$ & $(\mathrm{L} 1)(\mathrm{L} 2)$ & (L1) \\
\hline Est. 10 & 11 & 5 to & M & $(\mathrm{L} 1)(\mathrm{L} 2)$ & (L1) & (L1) \\
\hline
\end{tabular}

Nota: $(\mathrm{L} 1)$ = Aimara; $(\mathrm{L} 2)=$ Castellano; (L3) = Quechua.

\subsection{Resultados 2: Selección del Corpus, identificación de errores, clasificación,} explicación y conclusión

\section{Tabla 2}

Matriz de Análisis Contrastivo de Corder de la Estudiante $N^{\circ} 03$.

\begin{tabular}{|c|c|c|c|c|}
\hline $\begin{array}{l}\text { SELECCIÓN } \\
\text { DE CORPUS }\end{array}$ & $\begin{array}{l}\text { IDENTIFICACIÓN } \\
\text { DE ERRORES }\end{array}$ & CLASIFICACIÓN & EXPLICACIÓN & CONCLUSIÓN \\
\hline $\begin{array}{l}\text { Ficha de } \\
\text { desempeño de } \\
\text { competencias } \\
\text { lingüísticas en } \\
\text { la producción } \\
\text { de textos } \\
\text { escritos en } \\
\text { castellano. }\end{array}$ & $\begin{array}{l}\text { primito y yo a Iti a la } \\
\text { Picina en hay jugamos } \\
\text { y comemos. }\end{array}$ & $\begin{array}{l}\text { Trasferencia morfosintáctica } \\
\text { del aimara en la producción de } \\
\text { textos escritos en castellano. } \\
\text { Sujeto: primito y yo } \\
\text { Objeto: a Iti a la Picina en hay } \\
\text { Verbo: jugamos y comemos. }\end{array}$ & $\begin{array}{l}\text { La estudiante } \mathrm{N}^{\circ} 03 \text { hace uso de la estructura } \\
\text { morfosintáctica del aimara } \mathrm{S}+\mathrm{O}+\mathrm{V} \text { en la } \\
\text { producción de texto escrito en castellano. } \\
\text { Recordemos que: } \\
\text { La morfosintaxis del aimara es: }(\mathbf{S}+\mathrm{O}+\mathrm{V}) \\
\text { La morfosintaxis del castellano es: }(\mathbf{S}+\mathrm{V}+\mathrm{O}) \\
\text { Morfosintaxis del aimara } \mathrm{S+O}+\mathrm{V} \text { empleado en } \\
\text { el castellano. } \\
\text { "primito y yo a Iti a la Piscina en hay jugamos y } \\
\text { comemos" } \\
\text { Sujeto: primito y yo } \\
\text { Objeto: a Iti a la Picina en hay } \\
\text { Verbo: jugamos y comemos. } \\
\text { En la oración podemos apreciar que la estudiante } \\
\mathrm{N}^{\circ} 03 \text { hace uso de la morfosintaxis del aimara } \\
\text { S+O+V en la producción de textos escritos en } \\
\text { castellano, lo cual es incorrecto. } \\
\text { Traducción de la oración al aimara: (S+O+V) } \\
\text { Si traducimos esa oración al idioma aimara sería } \\
\text { de la siguiente manera: } \\
\text { "primitux nayampiw Itiru Piscinaru manqaptwa } \\
\text { ukamaraki anataptwa" }\end{array}$ & $\begin{array}{l}\text { En conclusión la } \\
\text { estudiante } \mathrm{N}^{\circ} 03 \text { hace uso } \\
\text { de la estructura } \\
\text { morfosintáctica de } \\
\text { aimara }(\mathrm{S}+\mathrm{O}+\mathrm{V}) \text { en la } \\
\text { producción de textos } \\
\text { escritos en castellano lo } \\
\text { cual es incorrecto para la } \\
\text { producción de textos } \\
\text { escritos en el idioma } \\
\text { castellano, puesto que la } \\
\text { estructura morfosintáctica } \\
\text { del castellano es } \\
(\mathbf{S}+\mathrm{V}+\mathrm{O}) \text {. }\end{array}$ \\
\hline
\end{tabular}


Si a la traducción empleamos la misma estructura morfosintáctica $\mathbf{S}+\mathrm{O}+\mathrm{V}$, sí es correcto porque esta estructura sí corresponde al idioma aimara.

"primitux nayampiw Itiru Piscinaru manqaptwa ukamaraki anataptwa"

Sujeto: primitux nayampiw

Objeto: Itiru piscinaru

Verbo: manqaptwa ukamaraki anataptwa.

Ficha de desempeño de competencias lingüísticas en la producción de textos orales en castellano.

\section{$[\ldots \ldots \ldots \ldots \ldots \ldots \ldots . .$.}

Entrevistador: $\quad Y a$,

¿qué has hecho en las

vacaciones?

Est. 03: Uhmm.. Ilo; eh

ido a Ilo

Entrevistador: Has ido

a Ilo, ¿qué más?

Est. 03: Hey ido a la piscina.

Entrevistador:

¿Luego?

Est. 03: Hemos,

hemos... No séee...ii

hemos comido, nos

hemos bañado, nos

hemos salido, estamos

muertos de sed, nos

hemos comprado.

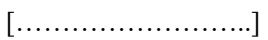

Falta de vocabulario en la producción de textos orales.
La estudiante $\mathrm{N}^{\circ} 03$ realiza el uso frecuente del término hemos; por lo que presenta dificultades en la producción de textos orales por la falta de vocabulario en la lengua castellana.
La estudiante $\mathrm{N}^{\circ} 03$ hace uso frecuente de los términos hemos; esto es probable a causa del poco dominio que presenta en la lengua castellana.

\section{Tabla 3}

Matriz de Análisis Contrastivo de Corder de la Estudiante $\mathrm{N}^{\circ} 09$

\begin{tabular}{lccc}
\hline SELECCIÓN & IDENTIFICACIÓN & CLASIFICACIÓN & EXPLICACIÓN
\end{tabular}

Ficha de yo Al serro fuí jugando.
desempeño de
competencias
lingüísticas en
la producción
de textos
escritos en
castellano.

\section{Trasferencia morfosintáctica del aimara en la producción de textos escritos en castellano.}

Sujeto: $y o$

Objeto: Al serro fuí

Verbo: jugando.
La estudiante $\mathrm{N}^{\circ} 09$ hace uso de la estructura morfosintáctica del aimara $\mathbf{S}+\mathrm{O}+\mathrm{V}$ en la producción de texto escrito en castellano.

\section{Recordemos que:}

La morfosintaxis del aimara es: $(\mathbf{S}+\mathrm{O}+\mathrm{V})$

La morfosintaxis del castellano es: $(\mathbf{S}+\mathrm{V}+\mathrm{O})$

Morfosintaxis del aimara $\mathrm{S}+\mathrm{O}+\mathrm{V}$ empleado en el castellano.

"yo Al serro fuí jugando"

Sujeto: $y o$

Objeto: Al serro fuí
En conclusión la estudiante $\mathrm{N}^{\circ} 09$ hace uso de la estructura morfosintáctica del aimara $(\mathbf{S}+\mathrm{O}+\mathrm{V})$ en la producción de textos escritos en castellano al igual que la estudiante $\mathrm{N}^{\circ}$ 03; lo cual es incorrecto para la producción de textos escritos en el idioma castellano, puesto que la estructura morfosintáctica del castellano es $(\mathbf{S}+\mathrm{V}+\mathbf{O})$. 
En la oración podemos apreciar que la estudiante $\mathrm{N}^{\circ} 09$ hace uso de la morfosintaxis del aimara $\mathrm{S}+\mathrm{O}+\mathrm{V}$ en la producción de textos escritos en castellano, lo cual es incorrecto.

Traducción de la oración al aimara: $(\mathrm{S}+\mathrm{O}+\mathrm{V})$ Si traducimos esa oración al idioma aimara sería de la siguiente manera:

"nayax qulluru sari anatampita"

$\mathrm{Si}$ a la traducción empleamos la misma estructura morfosintáctica $\mathbf{S}+\mathrm{O}+\mathrm{V}$, sí es correcto porque esta estructura sí corresponde al idioma aimara.

\section{"nayax qulluru sari anatampita"}

Sujeto: nayax

Objeto: qulluru sari

Verbo: anatampita.

\begin{tabular}{|c|c|c|c|}
\hline \multirow{4}{*}{$\begin{array}{l}\text { Ficha de } \\
\text { desempeño de } \\
\text { competencias } \\
\text { lingüísticas en } \\
\text { la producción } \\
\text { de textos orales } \\
\text { en castellano. }\end{array}$} & $\begin{array}{l}\text { Entrevistador: Hola, } \\
\text { ¿cuántos años tienes? }\end{array}$ & \multirow{4}{*}{$\begin{array}{l}\text { Préstamo linguístico del } \\
\text { idioma aimara en la } \\
\text { producción de textos orales. } \\
\text { Falta de vocabulario en la } \\
\text { producción de textos orales. }\end{array}$} & \multirow{4}{*}{$\begin{array}{l}\text { La estudiante } \mathrm{N}^{\circ} 09 \text { hace préstamo lingüístico de } \\
\text { una de las palabras del aimara en la producción } \\
\text { de textos orales en castellano; Según Bertonio } \\
\text { (1612) Waka significa vaca en el idioma aimara. } \\
\text { Waka = Vaca. } \\
\text { Así mismo realiza el uso frecuente del término } \\
\text { taba; por lo que presenta dificultades en la } \\
\text { producción de textos orales por la falta de } \\
\text { vocabulario en la lengua castellana. }\end{array}$} \\
\hline & Entrevistador: & & \\
\hline & $\begin{array}{l}\text { ¿qué has hecho en las } \\
\text { vacaciones? }\end{array}$ & & \\
\hline & Taba & & \\
\hline
\end{tabular}

La estudiante $\mathrm{N}^{\circ} 09$ realiza préstamo del término Waka del idioma aimara, hace uso frecuente de los término taba; esto es probable a causa del poco dominio que presenta en la lengua castellana.

pasteando mi waka.

Entrevistador: ¿Qué

más? ¿qué más has

hecho?

Est. 09: Taba jugando

voly.

Entrevistador: $\quad Y a$

¿qué más?

Est. 09: Taba lavando

mi ropa.

Entrevistador: $\quad Y a$

¿qué más has hecho?

Est. 09: Taba sacando

papa.

$[\ldots \ldots \ldots \ldots \ldots \ldots \ldots \ldots \ldots]$

\section{Tabla 4}

Matriz de Análisis Contrastivo de Corder de la Estudiante $\mathrm{N}^{\circ} 10$

\begin{tabular}{|c|c|c|c|c|}
\hline $\begin{array}{l}\text { SELECCIÓN } \\
\text { DE CORPUS }\end{array}$ & $\begin{array}{l}\text { IDENTIFICACIÓN } \\
\text { DE ERRORES }\end{array}$ & CLASIFICACIÓN & EXPLICACIÓN & CONCLUSIÓN \\
\hline
\end{tabular}




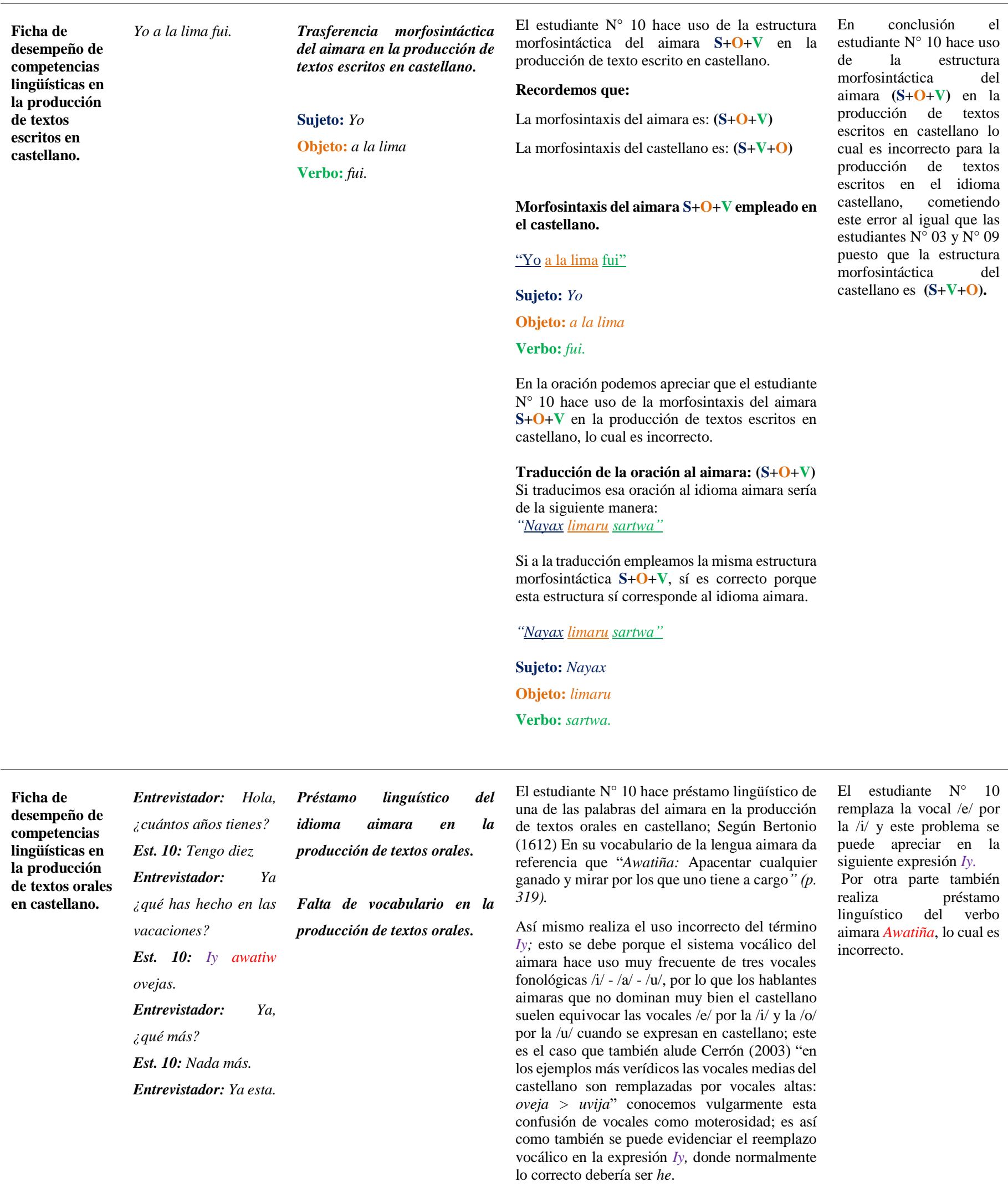

\section{Discusión}

Según nuestros resultados, existe transferencia morfosintáctica del aimara en el castellano en los estudiantes del quinto grado de la Institución Educativa Primaria N ${ }^{\circ} 70274$ Challapampa 
por lo que existe dificultades en el uso del castellano, estos resultados coinciden con la investigación desarrollada por Cortez (1999) quien también destacó que los estudiantes indígenas que ingresaron a la Universidad Mayor de San Marcos presentaron dificultades en el uso del castellano, tanto en forma oral como escrita.

La estudiante $\mathrm{N}^{\circ} 03$ hace uso de la estructura morfosintáctica del aimara $(\mathrm{S}+\mathrm{O}+\mathrm{V})$ en la producción de textos escritos en castellano lo cual es incorrecto para la producción de textos escritos en el idioma castellano, puesto que la estructura morfosintáctica del castellano es $(\mathrm{S}+\mathrm{V}+\mathrm{O})$, estos resultados coinciden con Merma (2007) quien manifiesta que "el castellano es una lengua flexible, y el aimara es una lengua aglutinante y, mientras que el castellano coloca el objeto de manera predominante después del verbo ( $\mathrm{S}$ V O), el aimara lo coloca antes ( $\mathrm{S} \mathrm{O}$ V). Desde el punto de vista sociolingüístico, el castellano y el aimara representan diferentes tipos de comunidades lingüísticas”. Así mismo la estudiante $\mathrm{N}^{\circ} 03$ hace uso frecuente del término "hemos" esto es probable a causa del poco dominio en la segunda lengua.

Por su parte la estudiante $\mathrm{N}^{\circ} 09$ también hace uso repetitivo del término “taba", Benavente (1985) refirió que observó un castellano motoso, donde se hace uso excesivo de diminutivos y el uso del elemento narrativo "dice". Por otra parte la estudiante $\mathrm{N}^{\circ} 09$ realiza préstamo lingüístico del término waka del idioma aimara.

Similares resultados halló Cerrón (2003) es por ello que tras su investigación manifiesta que en donde hay lenguas en contacto es normal encontrar problemas de trasferencia que afectan la performance de los bilingües; y tras esta investigación se pudo evidenciar que efectivamente se encontraron problemas de trasferencia y préstamos lingüísticos realizados como es el caso del estudiante $\mathrm{N}^{\circ} 10$ quien realizó el préstamo linguiístico awatiña del idioma aimara.

\section{Conclusión}

Una vez terminando el análisis de los textos escritos y orales que constituyen la muestra, se concluye lo siguiente.

La situación de lenguas en contacto que caracteriza al C.P. Challapampa, origina fenómenos que afectan a todos los niveles lingüísticos. En este caso, se ha observado que el aimara influye especialmente en el nivel morfosintáctico del castellano, donde los estudiantes hacen uso de la estructura morfosintáctica del aimara $\mathrm{S}+\mathrm{O}+\mathrm{V}$ propio del aimara para producir textos escritos en castellano, lo cual es incorrecto puesto que la estructura del castellano es $\mathrm{S}+\mathrm{V}+\mathrm{O}$.

En la producción de textos orales en castellano se evidenció el uso frecuente de expresiones como hemos y la expresión taba; así mismo se evidenció el uso de préstamos lingüísticos del 
idioma aimara como las expresiones waka, awatiw; por otra parte se pudo apreciar el remplazo de vocales como la /e/ por la /i/.

Este fenómeno linguiístico es un problema que sin duda afecta el rendimiento académico de los estudiantes, su autoestima e incluso su identidad lingüístico - cultural.

\section{Agradecimientos}

Agradecer a todos los miembros académicos y administrativos de la Institución Educativa Primaria $\mathrm{N}^{\circ} 70274$ Challapampa, por la acogida a dicha institución y brindarnos el acceso a investigar, y de manera muy especial a todos los estudiantes que participaron de dicha investigación, sin ellos no sería posible la culminación de este artículo de investigación.

\section{Referencias}

Benavente, I. (1985). El castellano en Puno: Variaciones sintácticas. Tesis de Maestría. Unidad de Maestría de la Universidad Nacional del Altiplano. Puno.

Bertonio, L. (1612). Trascripción del vocabulario de la lengua Aymara. Republicado por: Instituto de las Lenguas y Literaturas Andinas - Amazónicas. (ILLA - A).

Cerrón Palomino, R. (2003). Castellano andino. Cooperación Alemana al desarrollo. Primera edición. Lima, Perú.

Cortez, M. (1999). Características lingüísticas, culturales y educativas que presentan los estudiantes indígenas que ingresaron a la Universidad Mayor de San Marcos. Lima, Perú.

Godenzzi, J.C. (2005). En las redes del lenguaje: Cognición, Discurso y Sociedad en los Andes. Universidad del Pacífico. Centro de Investigación. Lima, Perú.

Granda, G. (1994). Español de América, español de África y hablas criollas hispánicas. Cambios, contacto y contexto. Madrid: Editorial Gredos.

Limo, A. y Salcedo, D. (2008). Contacto de lenguas: Una aproximación al caso del Perú y del español andino. ¿Cambio o muerte de las lenguas? Reflexiones sobre la diversidad, lingüística, social y cultural del Perú. Universidad Peruana de Ciencias Aplicadas. Lima, Perú. $127-142$.

Merma, G. (2007). Contacto lingüístico entre el español y el quechua: Un enfoque cognitivopragmático de las trasferencias morfosintácticas en el español andino peruano. Tesis doctoral para obtención del título de Doctor en Filología Española. Departamento de Filología Española. Universidad de Alicante, Alicante - España.

Miranda, L. (1998). La entrada del español en el Perú. Primera edición. Lima, Perú.

Montalvo, P. (2014). Trasferencia morfosintáctica del quechua (L1) en la producción de textos escritos (L2) en los estudiantes de la Institución Educativa Secundaria Agroindustrial de Cabanillas Lampa - Puno 2013. Tesis presentada como requisito para 
optar el título prefesional de Licenciado en Educación en la Univerdad Peruana Unión, Juliaca - Perú.

Real Academia Española (1998). El XXI Congreso de Academias de la Lengua Española. Los Ángeles.

Robledo, P. (2008). Culturas peruanas: Consecuencia del contacto en América. "Cambio o muerte de las lenguas”. Universidad Peruana de Ciencias Aplicadas. Lima, Perú.

Rodríguez, J. y Tovar, A. (1993). Análisis de errores originados por influencia de la pronunciación en las redacciones de estudiantes españoles de lengua inglesa. Valladolid, España.

Weinreich, U. (1974). Lenguas en contacto. Universidad Central de Venezuela. Venezuela.

Zimmermann, K. (2004). El contacto de las lenguas amerindias con el español en México. Revista Internacional de Lingüística Iberoamericana 2 - 2004. 19 - 39. 